\title{
Synthesis and Characterization of Hollow Mesoporous Nanometer Silica
}

\author{
Han Cui ${ }^{1, ~ a ~}$, Zhen Yu ${ }^{1, b}$, Mingguang Hu ${ }^{1, c}$, Liangchen Xing ${ }^{1, d}$,Jijun Xiao ${ }^{1, e, *}$ \\ ${ }^{1}$ School of Hebei University of Science \& Technology University, Heibei 050000, China; \\ acuihan18032831773@126.com, b1316080815@qq.com, '1297133041@qq.com, d961687775@qq. \\ com, jijunxiao@aliyun.com \\ *corresponding author
}

\begin{abstract}
In this study, Hollow-Mesopores $\mathrm{SiO}_{2}$ particles $\left(\mathrm{HM}-\mathrm{SiO}_{2}\right)$ were prepared. In preparation of $\mathrm{HM}-\mathrm{SiO}_{2}$, polystyrene (PS) particles with different sizes were prepared by emulsion polymerization method. The as-obtained PS particles were used as template, and then coated with silica by hydrolyisand condensation of tetraethoxy silane to obtain polystyrene/ $\mathrm{SiO}_{2}$ particles. Then hollow porous silica particles were obtained by treating core shell particles with tetrahydrofuran (THF) and acetone. The properties of products were characterized by FT-IR to characterize its structure. It showed that the obtained $\mathrm{SiO}_{2}$ nanoparticles with Hollow-Mesopores structure.
\end{abstract}

Keywords: Hollow, Mesoporous, Nanometer silica, Hard template.

\section{Introduction}

Hollow microspheres have multifunction properties comparing with single component microspheres.[1]Hollow microspheres materials, which could be obtained by removal of the core from core-shell microspheres, always have high surface area and low density, therefore it can be used as catalyst supports, adsorbent sensor and filler et.[2,3] TEOS was hydrolyzed in the synthesis mixture of PS particals directly by hard template method. Due to electrostatic interaction, the obtained $\mathrm{SiO}_{2}$ will be adsorbed on the PS particals surface to form microspheres.[4,5]

\section{Experimental}

\subsection{Materials}

2,2'-azobis(2-methylpropionamidine) dihydrochloride (AIBA) was provided by company of Qingdao RunXing Optoelectronic Materials Co., Ltd. Tetraethyl orthosilicate (TEOS) was supplied by Tianjin Kemiou Chemical Reagent Co., Ltd. Butyl acetate (BA) was supplied by Tianjin HengXing Chemical Prepiration Co.,Ltd. Polyvinylpyrrolidone (PVP) was purchased from Tianjin Daming Chemical Reagent Co.,Ltd. Styrene (St), 37\% wt hydrochloric acid ( $\mathrm{HCl})$, tetramethylene oxide (THF), acetone and anhydrous ethanol in analytical purity were obtained commercially andused as-received.

\subsection{Preparation of polystyrene hard template}

St, PVP and deionized H2O were first mixed in a round-bottom glass flask equipped with an electric stir. The mixture was made homogenous via stirring at a rate of $500 \mathrm{rpm}$ at room temperature for 30min. Then, AIBA was dissolved in water and added. The usage amount of PVP was varied at $8 \mathrm{~g}, 9 \mathrm{~g}, 10 \mathrm{~g}, 11 \mathrm{~g}, 12 \mathrm{~g}$. After $24 \mathrm{~h}$ of stirring at $75^{\circ} \mathrm{C}$, polystyrene emulsion with various particle sizes was obtained. The products were named PS-8, PS-9, PS-10, PS-11, PS-12. 


\subsection{Preparation of Hollow-Mesopores $\mathrm{SiO} 2$ particles}

Hollow-Mesopores $\mathrm{SiO} 2$ particles via the hydrolysis and condensation of TEOS. The $\mathrm{pH}$ of polystyrene emulsion having a particle size of about $100 \mathrm{~nm}$ was adjusted to 4 . Then, TEOS was dripped in the polystyrene emulsion. Then, two solutions were mixed and stirred for $24 \mathrm{~h}$ at $40^{\circ} \mathrm{C}$.

The white pristine PS-SiO2 powder was collected by removing the residual TEOS through three cycles of centrifugation/redispersion in anhydrous ethanol followed by removing the PS through added to THF and acetone respectively and stirred for $24 \mathrm{~h}$ at $55^{\circ} \mathrm{C}$. Hollow-Mesopores SiO2 particles was obtained.

\subsection{Characterization.}

Characterization FT-IR spectra of St and PS were recorded between $2000 \mathrm{~cm}-1$ and $500 \mathrm{~cm}-1$ on a Bruker Tensor-27 spec-trophotometer using $\mathrm{KBr}$ pellet technique. Morphological structures of pristine PS particles and HM-SiO2 were surveyed by a S-4800-I scanning electron microscope(SEM).

\section{Results and discussion}

Effect of PVP content on particle size of PS particles. In emulsion polymerization, the addition of PVP was used to adjust the particle size of the emulsion. Fig. 1 shows that polystyrene microspheres with different particle sizes can be obtained from different concentrations of PVP. With the dosage of PVP increasing from 8 to 12g, the particle size of the PS microsphere reduced from 250 to $130 \mathrm{~nm}$.

Structures and morphologies of $\mathrm{HM}-\mathrm{SiO} 2$. The $\mathrm{HM}-\mathrm{SiO} 2$ were prepared via reaction of TEOS hydrolysis. The chemical structures of St and obtained PS were detected by FT-IR spectra in Fig. 2 Because PVP C $=\mathrm{O}$ vibration peak at $1650 \mathrm{~cm}^{-1}$ (as shown in Fig. $2 \mathrm{a}$ and $2 \mathrm{~b}$ ) overlaps with the $\mathrm{C}=\mathrm{C}$ stretching vibration region, it is difficult to assign characteristic peaks at $1650 \mathrm{~cm}-1$ in Fig. 2a and Fig. $2 \mathrm{~b}$ to determine that polystyrene was synthesized. However, the absorption at $908 \mathrm{~cm}-1$ assigned to the deformation vibration weakens in Fig. 2a compared with Fig. 2b due to the styrene homopolymerization.

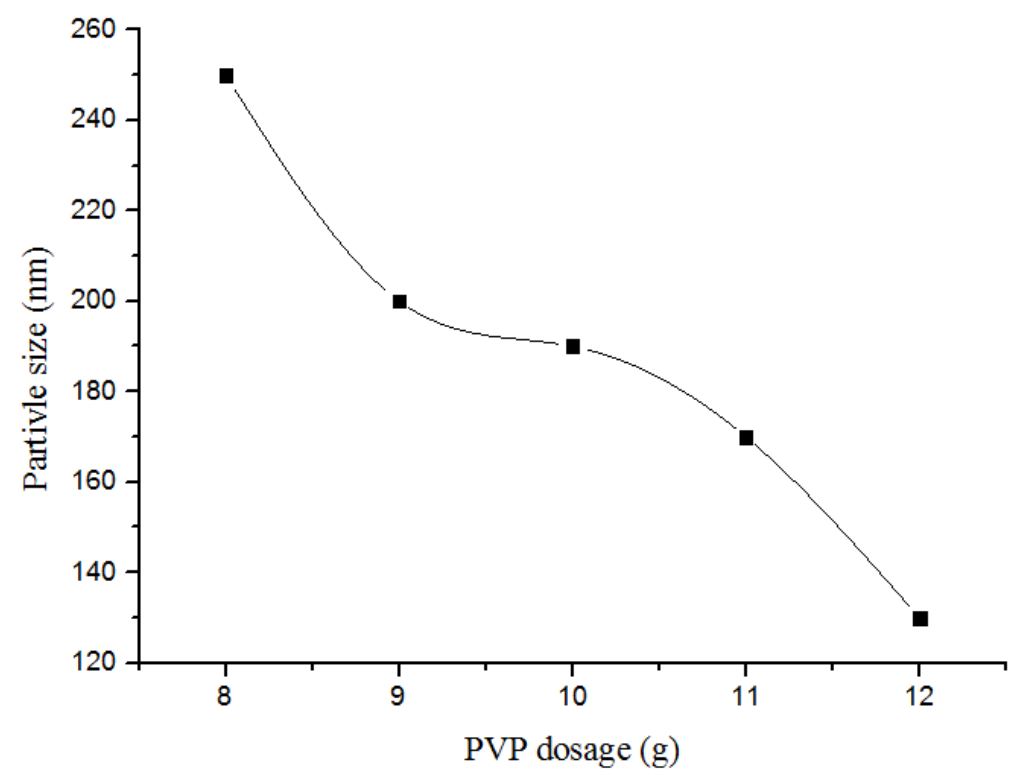

Fig. 1. Relationship between PVP content and particle size of PS microspheres 


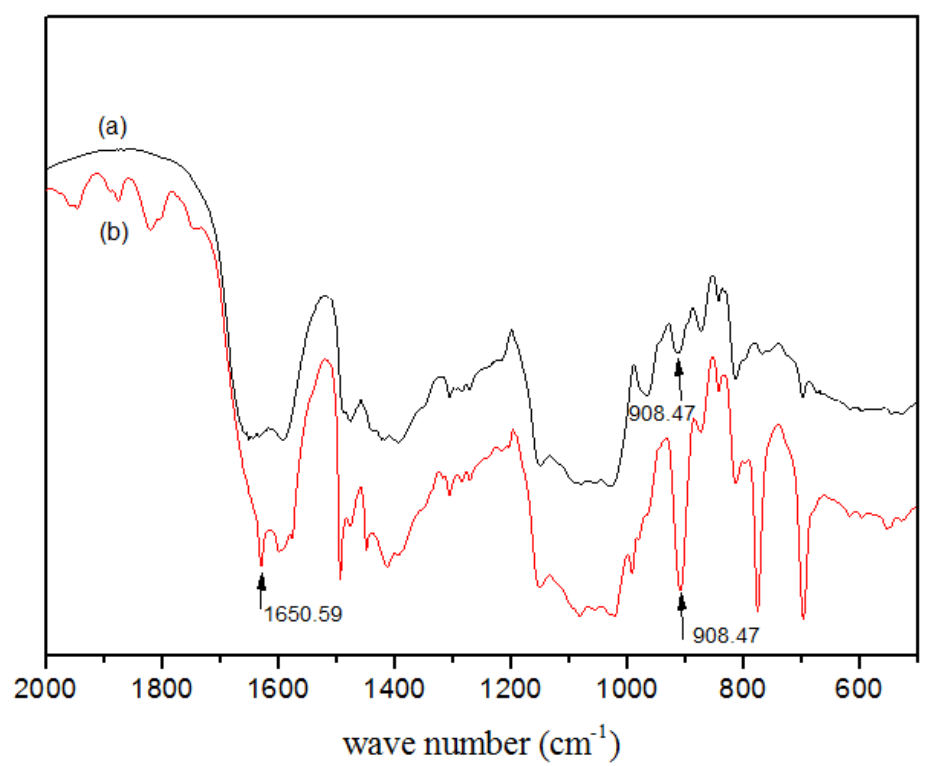

Fig. 2. FT-IR spectra:(a) PS (b) St

As indicated by SEM observation in Fig. 3a, the PS particles prepared in this work have an average diameter of ca.130nm. After the reaction of TEOS hydrolysis, the obtained $\mathrm{HM}-\mathrm{SiO}_{2}$ particles (Fig. 3b) have spherical shapes with hollow mesoporous structure and an average diameter of around 500nm.
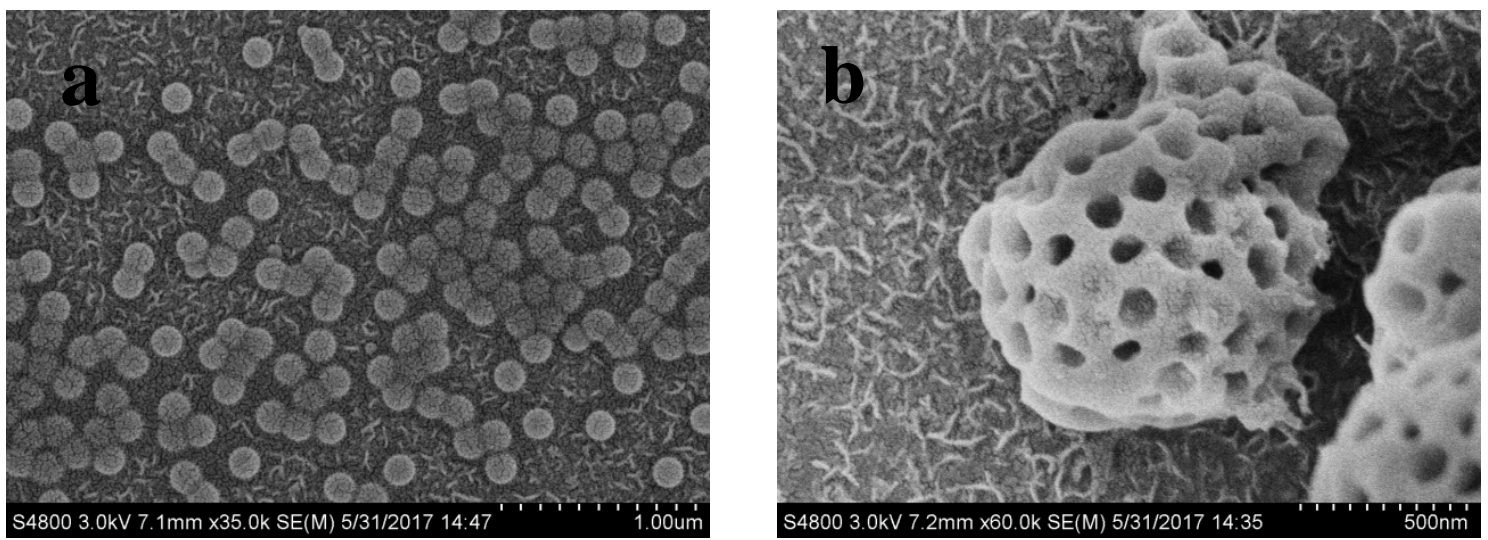

Fig. 3. SEM images: (a) PS particles (b) $\mathrm{HM}-\mathrm{SiO}_{2}$ particles

\section{Conclusions}

In the present work, size-controllable PS microspheres were prepared through emulsion polymerization. Hollow mesopores $\mathrm{SiO}_{2}$ microspheres can be obtained subsequently by acetone and THF washing PS-SiO 2 core-shell microspheres. The studied results indicated that PS microspheres was the template for hollow silica microspheres. Therefore, the particle size of the PS could affect the diameter of the hollow microspheres. The obtained $\mathrm{HM}-\mathrm{SiO}_{2}$ microspheres have particle size of about 500nm. Therefore, the method in this work has the potential to prepare various porous hollow silica microspheres .

\section{References}

[1] Minhua Ju , Yupeng Li , Liang Yu , Chongqing Wang, Lixiong Zhang,Preparation of size-controllable monodispersed carbon@silica core-shell microspheres and hollow silica microspheres,Microporous and Mesoporous Materials 247 (2017) 75-85. 
[2] I. Nongwe, V. Ravat, R. Meijboom, N.J. Coville, Pt supported nitrogen doped hollow carbon spheres for the catalysed reduction of cinnamaldehyde, Appl. Catal. A Gen. 517 (2016) 30-38.

[3] L. Guo, R. Zhang, C. Chen, J. Chen, X. Zhao, A. Chen, X. Liu, Y. Xiu, Z. Hou, Gold nanoparticles embedded in silica hollow nanospheres induced by compressed CO2 as an efficient catalyst for selective oxidation, Phys. Chem. Chem. Phys. 17 (2015) 6406-6414.

[4] W. Wang, M.-J. Zhang, L.-Y. Chu, Functional polymeric microparticles engineered from controllable microfluidic emulsions, Acc. Chem. Res. 47 (2014) 373-384.

[5] W. Liu, Y. Zhao, C. Zeng, C. Wang, C.A. Serra, L. Zhang, Microfluidic preparation of yolk/shell ZIF-8/alginate hybrid microcapsules from Pickering emulsion, Chem. Eng. J. 307 (2017) 408-417. 